\title{
Diffusion $k$-tensor Estimation from Q-ball Imaging Using Discretized Principal Axes
}

\author{
Ørjan Bergmann ${ }^{1,2}$, Gordon Kindlmann ${ }^{1}$, \\ Arvid Lundervold ${ }^{2}$, and Carl-Fredrik Westin ${ }^{1}$ \\ ${ }^{1}$ Laboratory of Mathematics in Imaging, Harvard Medical School, Boston MA, USA \\ ${ }^{2}$ University of Bergen, Postbox 7800, N-5020 Bergen, Norway
}

\begin{abstract}
A reoccurring theme in the diffusion tensor imaging literature is the per-voxel estimation of a symmetric $3 \times 3$ tensor describing the measured diffusion. In this work we attempt to generalize this approach by calculating 2 or 3 or up to $k$ diffusion tensors for each voxel. We show that our procedure can more accurately describe the diffusion particularly when crossing fibers or fiber-bundles are present in the datasets.
\end{abstract}

\section{Introduction}

Diffusion tensor Magnetic Resonance Imaging (DTI) has recently become an important tool in the analysis of the central nervous system. For quantitative analysis of possible white matter anomalies, parameters derived from the diffusion tensor can be associated with tissue microstructure and compared between health and disease. Diseases about which white matter abnormality is known or hypothesized (e.g. multiple sclerosis, Alzheimer's disease and schizophrenia) have successfully been investigated using a single diffusion tensor model 12 .

Upon inspection of histology, however, much of the white matter in primates has been shown to be composed of multiple interdigitating fibers. On the spatial scale of MRI, many voxels contain more than one fiber direction. The estimated diffusion tensor will then describe an average of the actual diffusion. Depending on the degree of intra-voxel heterogeneity, the single tensor fit may lead to inaccuracies in the diffusion tensor and derived quantities, such as fractional anisotropy (FA), linear, planar and spherical measures 3 and fiber trajectories.

In this work we have investigated multi-tensor estimation, in which we approximate the diffusion per voxel with more than one diffusion tensor. In particular, our approach allows the estimation of a user specified number $k$ tensors per voxel. We will show that our method for $k$-tensor estimation can better describe the actual diffusion in both real and synthetic datasets.

\subsection{Related Work}

In a related work Tuch [4] is able to make 2-tensor estimates by solving the multitensor model presented in section 2.1. In order to solve this set of equations, 
however, he assumes prior knowledge of the eigenvalues of the diffusion tensors about to be estimated. Jansons et al. 5] solves the same problem in the Fourier domain using a maximum-entropy parameterization, although their numerical approximations are sensitive to local minima, and are in general not guaranteed to converge to a global optimum. Peled et al. 6] use the coordinate frame of a single tensor fit to estimate two tensors residing in the resulting plane. It is not reported, however, if this approach can be generalized to $k$ larger than 2 .

\section{Background}

In diffusion-weighted imaging, the image contrast is related to the local mobility of water molecules in living tissue, where the measurements can be made sensitive to water self-diffusion along $n$ distinct spatial directions $\mathbf{g}_{1}, \cdots, \mathbf{g}_{n}$, specified by the image acquisition protocol, such that for each voxel we obtain a collection of $n$ measurements $S_{1}, \cdots, S_{n}$.

These measured values $S_{i}$ are related to the $3 \times 3$ diffusion tensor $X$ as described by the Stejskal-Tanner equations 3 .

$$
S_{i}=S_{0} \exp \left(-b \mathbf{g}_{i}^{T} \mathbf{X} \mathbf{g}_{i}\right)
$$

where $b$ is an acquisition-specific constant, $S_{0}$ is signal intensity without diffusion sensitization, and unit-length gradient directions $\mathbf{g}_{1}, \cdots, \mathbf{g}_{n}$. The number of equations $n$ is usually larger than the minimum 6 required for a single tensor estimate, so least-squares minimization is used.

By taking the logarithm and rearranging, the estimation of the symmetric $3 \times 3$ diffusion tensor $\mathbf{X}$ can be found by solving the linear least-squares problem

$$
\min _{\mathbf{X}} \sum_{i}\left(\mathbf{g}_{i}^{T} \mathbf{X} \mathbf{g}_{i}-d_{i}\right)^{2}
$$

where

$$
d_{i}=\frac{\log \left(S_{0}\right)-\log \left(S_{i}\right)}{b}
$$

These $d_{i}$ values are in the literature commonly referred to as the apparent diffusion coefficients (ADC), and we will let the term ADC profile denote the surface spanned by the $d_{i} \mathbf{g}_{i}$.

One common way to visualize a diffusion tensor is by the letting the eigenvectors define the orientation of an ellipsoid, with per-axis scaling determined by the corresponding eigenvalues. Figure 1 shows an estimated diffusion tensor and the corresponding ADC profile for a synthetic example. We observe that the orientation of the ADC profile seems to correspond well with the orientation of the diffusion tensor in that they both have their longest extent along the $x$-axis.

\subsection{Multi-Tensor Model}

Equation (1) describes the relationship between one diffusion tensor $\mathbf{X}$ and the measured MRI signals $S_{i}$. However, sometimes the measured $S$-values are not 


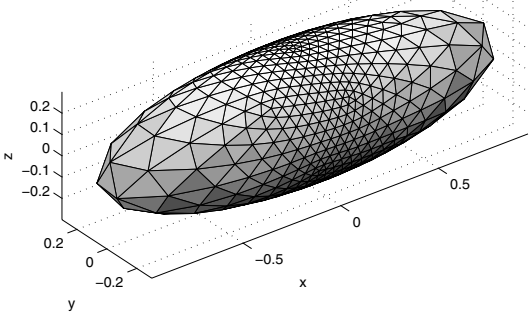

(a) The diffusion tensor

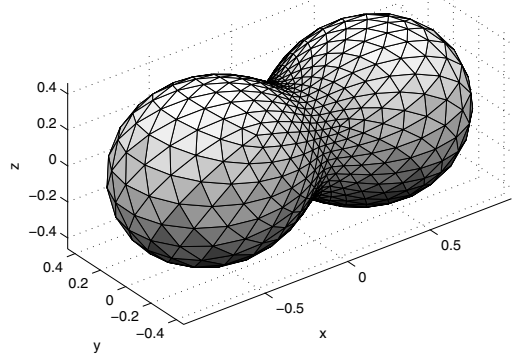

(b) The corresponding ADC profile

Fig. 1. A synthetic diffusion tensor with principal diffusion along the $x$-axis and with eigenvalues $\lambda=\left\{1, \frac{1}{3}, \frac{1}{3}\right\}$, with $b=700, S_{0}=1$, using $n=642$ uniformly distributed gradient directions

well modeled by a single diffusion tensor, due to a more heterogeneous distribution of fiber orientations, as arises with crossing fibers.

To cope with this situation, Tuch [4 has modeled the relationship between the $k$ tensors $\mathbf{X}_{j}$ and the measured $S_{i}$ values as

$$
S_{i}=S_{0} \sum_{j=1}^{k} f_{j} \exp \left(-b \mathbf{g}_{i}^{T} \mathbf{X}_{j} \mathbf{g}_{i}\right)
$$

where $f_{j}$ are non-negative weights which sum to 1 for $j=1, \cdots, k$ and $i=$ $1, \cdots, n$. Note that this model reduces to that of (1) when $k=1$.

Fig. 2 shows a synthetic example with $k=2$. $S$-values calculated according to (3) produce the ADC profile in Fig. 2(b) Note the apparent $45^{\circ}$ shift of the main axes of the ADC profile relative to the orientation of the original tensors. As the angle between the two synthetic tensors varies, the relative size and angle between the two main extent directions of the ADC profile changes. This nonintuitive behavior motivates a different diffusion description, the Q-ball.

\subsection{Approximating the Q-ball}

The basic idea of Q-ball imaging [47] is to transform the measured diffusion signal to a orientation distribution function directly on the sphere. Considering each point on the sphere as a pole, the Q-ball transform assigns the value at the pole to be the integral over the associated equator.

In our implementation we approximate the Q-ball by taking a given $\mathbf{g}_{i}$ as a plane normal, and let the associated Q-ball value $q_{i}$ be a weighted sum of $S_{j^{-}}$ values where the weights associated with $S_{j}$ are inversely proportional to the distance from $\mathbf{g}_{j}$ to the plane. Specifically,

$$
q_{i}=\frac{1}{S_{0}} \sum_{j=1}^{n} S_{j} \cos \left(\frac{\pi}{2} \mathbf{g}_{i}^{T} \mathbf{g}_{j}\right)^{p}
$$




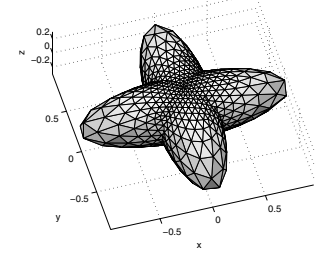

(a) Two diffusion tensors

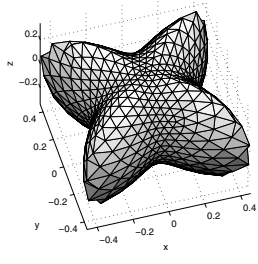

(b) ADC profile

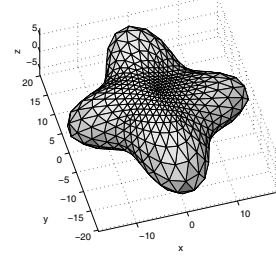

(c) Q-ball profile

Fig. 2. $k=2$ synthetic diffusion tensors with principal diffusion along the $x$-axis and $y$-axis, respectively, and both with eigenvalues $\lambda=\left\{1, \frac{1}{3}, \frac{1}{3}\right\}$. We let $f=\left\{\frac{1}{2}, \frac{1}{2}\right\}$ and selected the other parameters as in Fig. 1

where $p$ is a constant, in the current implementation $p=5$. We define the Q-ball profile as the surface spanned by $q_{i} \mathbf{g}_{i}$, shown in Fig. 2(c). Observe that the Qball profile extrema now correspond better with the extremes of the two original tensors.

\section{Methods}

We estimate $k$ diffusion tensors by segmenting the $S$-values. To simplify our notation, we identify each of the values $S_{i}$ simply by its index $i$. We segment these $n$ values into $k$ disjoint sets $Z_{l}$ for $l \in 1,2, \cdots, k$. A diffusion tensor $\mathbf{X}_{l}$ is then estimated from each set $Z_{l}$, using (2) with $i \in Z_{l}$. Note that when $k=1$, all samples are segmented into the same set $Z_{1}=\{1,2, \cdots, n\}$, and the approach reduces to the standard single tensor estimation (Section 2 ).

\subsection{Segmentation}

The Q-ball profiles is used to segment the samples. Each triangle vertex in Fig. 2(c) corresponds to one $q_{i} \mathbf{g}_{i}$, calculated from the sample $i$. Thus segmentation of the Q-ball vertices corresponds to a segmentation of the original samples.

The vertices of the Q-ball can naturally be segmented into $k=2$ sets in Fig. $2(\mathrm{c})$ one belonging to each of the two principal (directions, extents, or simply) axes of the Q-ball. In the following we define an axis $j$ to be a line going through the origin and a unique point $p_{j}$ taken from a uniformly distributed set of points on the unit sphere. However, since we do not know in advance what the principal axes are, we make the following simplification: we will only consider $m$ discretely sampled axes in our approach, and attempt to choose the $k$ which matches the vertices best. We will then attempt to pair each of the vertex points (identified by the index $i$ ) with exactly one of the $k$ axes.

Assigning a vertex $i$ to axis $j$ will contribute an error (or cost) $c_{i j} \geq 0$, and we define this error to be the shortest distance from vertex $i$ to axis $j$. Orthogonality conditions give

$$
c_{i j}=\left\|p_{j} \times\left(-q_{i} \mathbf{g}_{i}\right)\right\|_{2}
$$


where $\times$ denotes vector cross-product. The assignment of vertices to axes should minimize the total cost of the assignments.

We can now formulate the problem of assigning vertices to axes as a binary integer program (BIP). In order to do so we first define our variables. We let

$$
x_{i j}= \begin{cases}1, & \text { iff vertex } i \text { is assigned to axis } j \\ 0, & \text { otherwise }\end{cases}
$$

and also define

$$
y_{j}= \begin{cases}1, & \text { iff any vertex has been assigned to axis } j \\ 0, & \text { otherwise }\end{cases}
$$

Having done so we write the following minimization problem

$$
\begin{gathered}
\min _{x_{i j}} \sum_{i} \sum_{j} c_{i j} x_{i j} \\
\text { s.t. } \sum_{j} x_{i j}=1 \\
x_{i j} \leq y_{j} \\
\sum_{j} y_{j}=k \\
x_{i j} \in\{0,1\}, y_{j} \in\{0,1\}
\end{gathered}
$$

where $i=1,2, \cdots, n$ and $j=1,2, \cdots, m$. Here (5) ensures that each vertex is assigned to exactly one axis. Equation (6) ensures that if any vertex is assigned to axis $j$, then axis $j$ is labeled as "in use". Finally, (7) ensures that exactly $k$ out of the $m$ axes are in use.

One important observation learned from our BIP formulation is that once the choice of which axes to use has been made (that is, the variables $y_{j}$ has been chosen) the remaining variables can be selected in a straightforward manner: Let $J$ denote the set $\left\{j: y_{j}=1\right\}$. Then it is optimal to let

$$
x_{i j}= \begin{cases}1, & \text { iff } c_{i j} \text { is the smallest value in the set }\left\{c_{i p}: p \in J\right\} \\ 0, & \text { otherwise }\end{cases}
$$

for all $i$ when $j \in J$. For all other $i$ and $j, x_{i j}=0$.

When $k$ is small, this observation suggests that we can solve the BIP by exhaustion; enumerate all the possible choices of $y$, select $x$ as suggested above and save the solution which has the minimum objective-function value.

\section{Results}

Figure 3 shows results a synthetic test case which varies the angle between the originating principal diffusion directions. The estimated diffusion tensors 

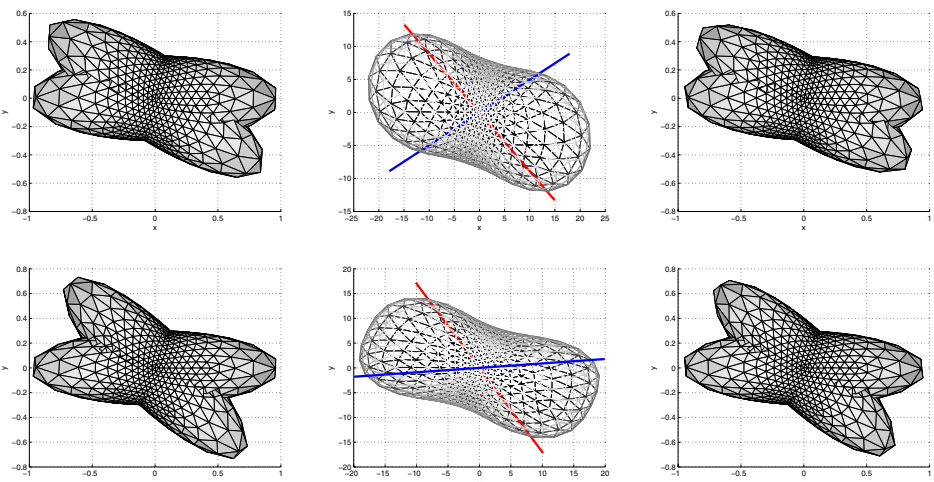

Fig. 3. The leftmost column shows $k=2$ synthetic diffusion tensors with principal diffusion directions $30^{\circ}$ and $45^{\circ}$ apart. The middle column shows the Q-ball profile and the estimated principal axes. The last column shows the estimated diffusion tensors.

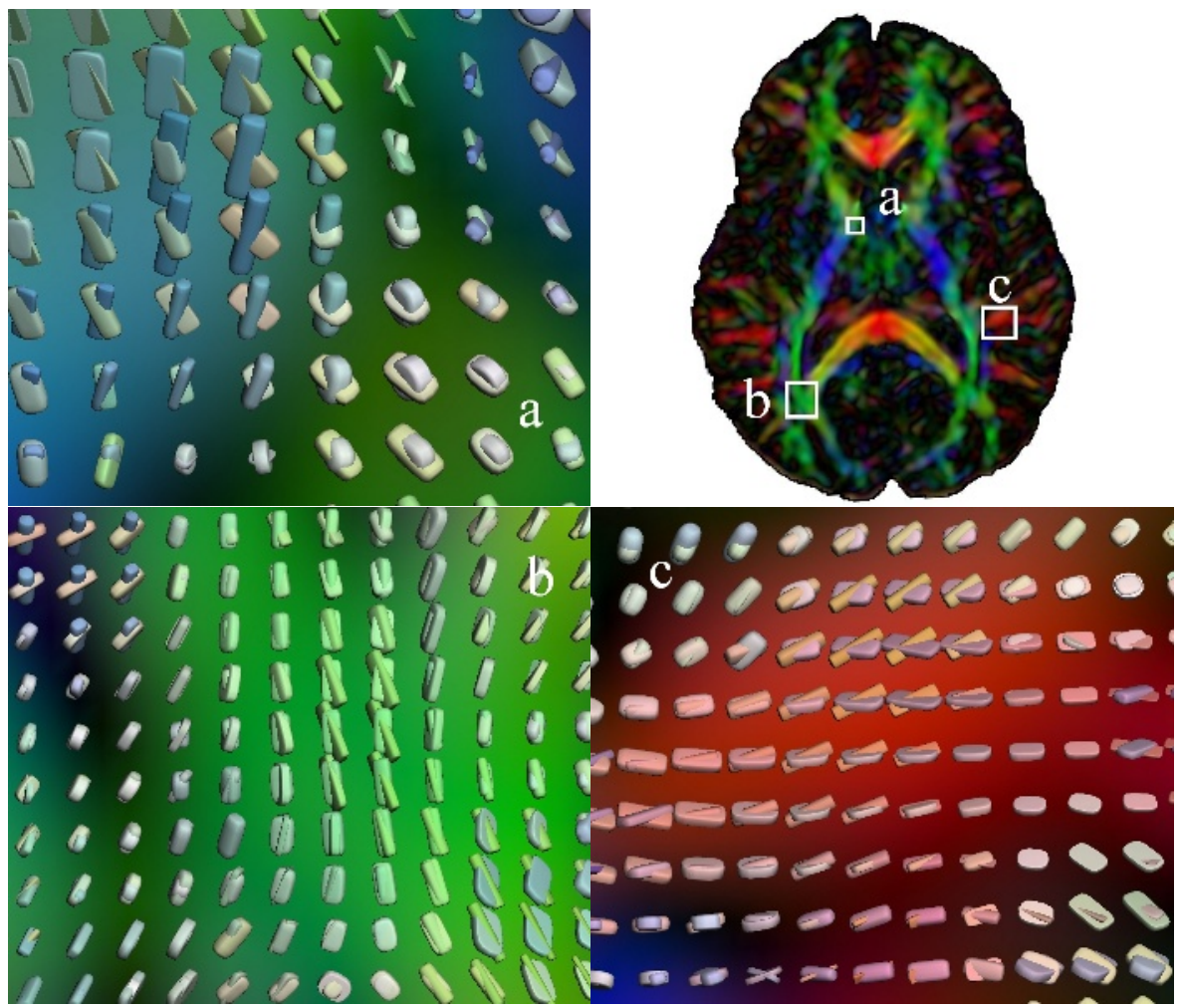

Fig. 4. Two tensor approximation in each voxel of a real DTI dataset. The upper right hand panel shows the position of the regions (a)-(c) visualized in the upper left, lower left and lower right panels, respectively. 
correspond well with the original ones. Specifically, the sum of error norms $\sum_{l=1}^{k}\left\|\mathbf{X}_{l}^{\text {original }}-\mathbf{X}_{l}^{\text {estimated }}\right\|_{F}$ for the $30^{\circ}$ and $45^{\circ}$ cases are $8.24 \times 10^{-2}$ and $5.45 \times 10^{-2}$, respectively, letting other parameters be as in Fig. 2 .

Using the same setup, our algorithm was unsuccessful in computing two tensors when the angle between principal diffusion directions became smaller than $30^{\circ}$. One of the estimated principal axes became orthogonal to the $x y$-plane, resulting in a bad segmentation. However, as the angle between the two principle diffusion directions decreases, the single tensor fit in fact becomes more appropriate, so we currently have not made attempts to alleviate this problem.

Figure 4 shows an axial slice from a healthy human volunteer DTI scar 1 . Two tensors were approximated for each voxel and their estimates were visualized as described in $[8$. The background images are direction-encoded FA maps where red indicates right-left, green indicates anterior-posterior and blue indicates a superior-inferior primary diffusion direction of the single tensor fit.

We observe spatially consistent orientations of two diffusion directions that are plausible given the known predominant fiber directions in these regions.

\section{Discussion}

In this work we have presented a novel way to estimate several diffusion tensors per voxel. To implement this approach no extra data needs to be acquired other than what is routinely available from clinical scans. This fact enables larger clinical studies to be performed with already existing DTI datasets, although we have not attempted to do so thus far. We also point out that since we rely on Q-ball imaging, we expect the procedure to give better results when many gradient diffusion directions are used in the acquisitions.

Our approach differs from those outlined in [46] in that we do not explicitly try to solve (3). Therefore we do not get the weighting parameters $f_{j}$ of that model either, however by substituting our estimated tensors into (3), these values can be estimated by solving the resulting constrained linear problem.

Deciding how many tensors one should try to fit to the data is in itself a difficult problem. As [46] points out, there is some evidence to suggest that 2 tensors might be appropriate when an estimated single tensor is planar in form. One measure of this oblateness is when the difference between the second and the third largest eigenvalues of the tensor, i.e. $\lambda_{2}-\lambda_{3}$, is large. Ultimately, the choice of the parameter $k$ depends on what's being studied and we have not considered how to choose it as a part of this work.

Other segmentation strategies based on the Q-ball profile are possible, such as $k$-means clustering. This is potentially faster, but $k$-means clustering does not in general guarantee optimality of the final segmentation, given its dependence on an initial (often random) configuration. Since our current focus has been to ensure a good segmentation rather than than a fast one, we have not considered $k$-means clustering any further, but hope to do so in the future.

${ }^{1}$ DTI data was estimated from 30 DWIs at $\mathrm{b}=700 \mathrm{~s} / \mathrm{mm}^{2}$ and 5 non-DWI T2s, from a 1.5 T Philips scanner, with resolution $0.94 \times 0.94 \times 2.5 \mathrm{~mm}$. 


\section{Acknowledgments}

This work supported by NIH NIBIB T32-EB002177 and NIH NCRR P41-RR13218 (NAC). We also acknowledge support from the Norwegian Research Council (ISBILAT). DWI data courtesy of Dr. Susumu Mori, Johns Hopkins University, supported by NIH R01-AG20012-01 and P41-RR15241-01A1.

\section{References}

1. Ramnani, N., Behrens, T., Penny, W., Matthews, P.: New approaches for exploring anatomical and functional connectivity in human brain. Biological Psychiatry 56(9) (2004) 613-619

2. Sundgren, P., Dong, Q., Gomez-Hassan, D., Mukherji, S., Maly, P., Welsh, R.: Diffusion tensor imaging of the brain: Review of clinical applications. Neuroradiology 46(5) (2004) 339-350

3. Westin, C.F., Maier, S., Mamata, H., Nabavi, A., Jolesz, F.A., Kikinis, R.: Processing and visualization for diffusion tensor MRI. Medical Image Analysis 6 (2002) 93-108

4. Tuch, D.S.: Diffusion MRI of Complex Tissue Structure. PhD thesis, Massachusetts Institute of Technology (2002)

5. Jansons, K., Alexander, D.: Persistent angular structure: New insights from diffusion magnetic resonance imaging data. Inverse Problems 19 (2003) 1031-1046

6. Peled, S., Westin, C.F.: Geometric extraction of two crossing tracts in DWI. In: Proceedings of 13th ISMRM. (2005)

7. Tuch, D.S.: Q-ball imaging. Magnetic Resonance in Medicine 52 (2004) 1358-1371

8. Kindlmann, G.: Superquadratic tensor glyphs, School of Computing, University of Utah, USA, Joint Eurographics - IEEE TCVG Symposium on Visualization, The Eurographics Association 2004 (2004) 